\title{
Changes in plasma lipid and in-hospital deaths in patients with sepsis
}

\author{
Azam Nabavi ${ }^{1}$, Abbas Allami*1(D), Reza QasemiBarqi $^{1}$ \\ Received: 30 Dec 2018 \\ Published: 9 May 2020
}

\begin{abstract}
Background: Lipid profiles are infrequently measured in clinical management of sepsis patients. Sepsis leads to significant alterations in the metabolism of lipids. The aim of the present study was to determine whether changes in plasma lipid concentrations during sepsis treatment were associated with clinical outcome.

Methods: In this study, 74 adult septic patients were included in this prospective observational study from January to December 2017. Patients taking lipid lowering agents were excluded. A detailed medical history was obtained and clinical examination was performed. Serum total cholesterol (STC) and its fractions [low-and high-density lipoprotein] and triglyceride levels were measured in the morning of the first day after admission and then once weekly. The primary outcomes of the study were in-hospital mortality, and hospital stay and hypocholesterolemia were defined as STC levels $<50 \mathrm{mg} / \mathrm{dL}$. Manne-Whitney U and chi-squared tests were used for data analysis, and significance level was set at $\mathrm{p}<0.05$.

Results: In this study, 78.4\% (CI 95\%: 67.3-87.1) of patients had hypocholesterolemia. During the study period, 21.6\% (CI 95\%: 12.9- 32.7) of patients died. All lipid (except TG) concentrations continuously decreased in deceased sepsis patients but increased in recovering patient ( $\mathrm{p}$ value for STC $(\mathrm{p}=0.004)$, LDL $(\mathrm{p}=0.006)$, HDL $(\mathrm{p}=0.010)$, and TG $(\mathrm{p}=0.052))$. The serum lipids concentration was not associated with length of hospital stay ( $p$ value for STC $(p=0.524)$, LDL $(p=0.813)$, HDL $(p=0.799)$ and TG $(p=0.581)$ ).

Conclusion: In this study it was found that the additional decline of lipid profile was significantly associated with increased mortality rate of sepsis patients. Thus, the clinically termed 'the lipaemia of sepsis' is not true in all situations.
\end{abstract}

Keywords: Cholesterol, Lipoprotein, LDL, HDL, Triglyceride, Sepsis, Emergency service, Hospital

Conflicts of Interest: None declared

Funding: The present study has been financially supported by the research deputy of Qazvin University of Medical Sciences (project number: 14002138).

\section{*This work has been published under CC BY-NC-SA 1.0 license.}

Copyright $₫$ Iran University of Medical Sciences

Cite this article as: Nabavi A, Allami A, QasemiBarqi R. Changes in plasma lipid and in-hospital deaths in patients with sepsis. Med J Islam Repub Iran. 2020 (9 May);34:45. https://doi.org/10.47176/mjiri.34.45

\section{Introduction}

Sepsis is a frequent cause of admission and a frequent fatal condition characterized by a dysregulated inflamma-

tory response to microbial infection and altered metabolic state, leading to tissue injury and organ failure (1). Several
Corresponding author: Dr Abbas Allami, allami@qums.ac.ir

Department of Infectious Diseases, Qazvin University of Medical Sciences, Qazvin, Iran $\uparrow$ What is "already known" in this topic:

Lipid profiles are infrequently measured in clinical management of sepsis patients. The majority of previous studies focused on the comparative analysis of mean values of serum lipids between survivor and nonsurvivor groups or between sepsis and severe sepsis groups.

$\rightarrow$ What this article adds:

In this study, serum lipid levels were analysed over time and the association between lipid levels and survival in patients with sepsis was examined. The prevalence of hypocholesterolemia in sepsis patients was significantly higher than in the general population. However, absolute STC, LDL, HDL, and TG levels at the time of admission was not suitable to predict mortality in sepsis patients. The data of this study support the hypothesis that higher decline of serum total cholesterol and other lipid profile during hospital stay are associated with a higher mortality in sepsis patients. Thus, the clinically termed 'the lipaemia of sepsis' is not true in all situations. 
reports have revealed the incidence of sepsis and severe sepsis increasing in excess of the growth of the population (2). Many studies showed dramatic lipid profile changes when individuals became ill $(3,4)$. These alterations have been described in patients with a wide range of disorders, including infection (4). During the course of infection, significant changes are observed in patients' serum profile of lipids (5). Early reports have described a cytokineinduced hyperlipoproteinaemia, the so called "lipaemia of sepsis", which was originally thought to represent the mobilization of lipid stores, especially serum total cholesterol and triglyceride $(6,7)$. However, some studies have challenged this concept in the presence of severe infection and indicated that total and high-density lipoprotein cholesterol (HDL-C) levels are commonly decreased, with varying changes in triglyceride (TG) levels in sepsis; they also noted that the magnitude of the changes seems to reflect the severity of inflammation $(6,8,9)$. Lipid profiles are rarely measured or interpreted in clinical management of sepsis and are not mentioned in the Surviving Sepsis Campaign guidelines $(3,10)$. Thus, this longitudinal study aimed to determine whether change in plasma lipid concentrations during sepsis treatment were associated with clinical outcome (death and duration of admission).

\section{Methods \\ Setting}

This prospective observational study was performed at Bou-Ali University Hospital, a 250-bed tertiary center with a referral population of 1270000 in Qazvin province, northern Iran. The study participants were recruited from the sepsis patients who referred to the Emergency Department (ED) from January to December 2017.

\section{Patients}

Informed consent was obtained from all participants and their caregivers. A total of 74 adult sepsis patients (aged 18 years or older) who referred to the ED were enrolled. Patients were interviewed at the ED or within the first 24 hours after hospital admission. The following data were gathered: smoking status; sex; age comorbidities abstracted from the patient history including hypertension, diabetes mellitus (DM), ischemic heart disease, neurologic disease (cerebrovascular accident (CVA) and dementia, renal failure, and chronic obstructive pulmonary disease. Detailed medical history was obtained and clinical examination performed. Patients taking lipid lowering agents were excluded. During the hospital stay, information on the final diagnosis and therapeutic procedures was recorded, including starting and ending dates. The criteria for including the patients into the case group were having 2 to 4 of the following signs, which are considered as septic: hypo or hyperthermia (temperature $<36$ or $>38^{\circ} \mathrm{C}$ ), tachycardia (pulse rate of 90 or more per minute), tachypnea (respiratory rate (RR) above 20 per minute), increased or decreased total white blood cells (WBC) counts (WBC $>12000$ or $<4000$ ), and identification of an infection site. The term sepsis applies to patients who meet Systemic Inflammatory Response Syndrome (SIRS) criteria in response to a known source of infection (11). Information on variables associated with sepsis (eg, microorganism in blood culture) was also gathered. The severity of sepsis was classified according to the criteria proposed by the Third International Consensus Definitions for Sepsis and Septic Shock as death anticipated during hospitalization (12).

\section{Blood sampling and laboratory methods}

Serum samples were obtained from whole blood samples collected in tubes without anticoagulant and analyzed for C-reactive protein (CRP), erythrocyte sedimentation rate (ESR), WBC (and poly morphonuclears [PMN]\%), blood sugar $(\mathrm{BS})$, and creatinine $(\mathrm{Cr})$ by conventional methods. Serum total cholesterol (STC) level and its fractions [levels of low-density lipoprotein cholesterol (LDLC) and HDL-C] and TG levels were measured in the first morning after admission and then once weekly. Serum samples were analyzed immediately or stored at $4^{\circ} \mathrm{C}$ and analyzed within 12 hours of collection at the Bou-Ali hospital laboratory using standardized enzymatic methods. All samples were analyzed in the same laboratory. To ensure the reliability and validity of all biochemical measures, $32.4 \%$ of samples were rechecked. Also, arterial blood samples were obtained to measure arterial blood gas (ABG) values (bicarbonate (HCO3-) and Pao2/Fio2) in the initial emergency department (ED) evaluation of patients. Blood cultures were obtained before initiating antimicrobial therapy. Hypocholesterolemia and hypotriglyceridemia were defined as $\mathrm{TC}<150 \mathrm{mg} / \mathrm{dL}$ and $\mathrm{TG}<50 \mathrm{mg} / \mathrm{dL}$, respectively $(13,14)$. For analytic purposes, 3 categories of STC levels were defined: $<150$ $\mathrm{mg} / \mathrm{dL}$ (hypocholesterolemia), 150 to $199 \mathrm{mg} / \mathrm{dL}$ (normal cholesterol levels), and $>200 \mathrm{mg} / \mathrm{dL}$ (hypercholesterolemia). Erythrocyte sedimentation rate was measured by the Westergren method. The secondary endpoint was to assess differences in hospital length of stay. The study was approved by the ethics committee of Qazvin University of Medical Sciences (ethical approval number: IR.QUMS.REC.1395.265).

\section{Statistical analysis}

A sample size was calculated based on published data of an earlier study, in which plasma lipid levels with the exception of TG were significantly lower in patients who developed multiorgan dysfunction syndrome (MODS) compared to those who did not (total cholesterol: $91.4 \pm 45.0$ vs. $129.9 \pm 43.7$ ) (9). Also, it was estimated that 74 patients were needed to detect a mean difference $38 \mathrm{mg}$ $\mathrm{mg} / \mathrm{dL}$ between primary STC, with a power of $80 \%$ and an alpha level of 0.05. Data were analyzed using SPSS 22.0 (IBM Inc., USA). The primary independent variable was TC and its fractions and TG; the secondary independent variables were age, sex, comorbidities, prescribed medications, and biochemical variables, and the primary outcome variable was survival. Distributions of continuous data were assessed for normality using the Kolmogorove Smirnov test. Continuous variables were described as mean \pm SD or median (interquartile range) and were compared using $\mathrm{t}$ test for independent samples or ManneWhitney $U$ as appropriate. Categorical variables were de- 
scribed as percent of indicator value and compared by vital status using chi-squared test or the Fisher's exact test as appropriate. All tests were 2-sided and considered significant at $\mathrm{p}<0.05$. Prevalence of hypocholesterolemia in sepsis patients was compared with Iranian general population. In this study, serum lipid profile levels $(\mathrm{mg} / \mathrm{dL})$ were divided into 5 groups by age, 2 groups by sex (male, female and total), and 7 groups by percentiles of lipid level (5th, 10th, 25th, 50th, 75th, 90th, 95th). Overall, $8 \%$ of the population had STC levels below $150 \mathrm{mg} / \mathrm{dL}$ (15).

\section{Results}

\section{Population}

The mean \pm SD of patients' age was $65.1 \pm 18.6$ years, which varied from 21 to 92 years (median 68 year). The female to male ratio was $0.85 ; 54.1 \%$ of the participants $(\mathrm{n}=40)$ were male. During the study period, $21.6 \%$ (CI $95 \%$ : $12.9-32.7)$ of patients $(n=16)$ died and $78.4 \%$ were discharged from the hospital. Only 7 patients were hospitalized for longer than 3 weeks. Characteristics of the study population are presented in Table 1 .

Participants who survived to the end of follow-up were significantly younger $(\mathrm{p}=0.025)$. Also, qSOFA score was elevated in the nonsurvivor group and odds ratio for qSOFA (low risk / high risk) was 22.83 (CI 95\%: 2.82184.82).

A positive blood culture result was obtained in $21.6 \%$ of the patients. The percentage of patients that survived was not different for those with a positive blood culture result $(17.2 \%)$ than for those that had a negative culture $(37.5 \%)$. In this study, $17.6 \%$ positive cultures were Gram-negative infections (the most common: pseudomonas and E. coli) and 5.4\% of cultures yielded Grampositive organisms. In addition, bicarbonate levels were significantly lower in survivors, but no significant differences were observed for other clinical parameters (eg, CRP, ESR, and $\mathrm{Cr}$ ).

\section{On admission lipid profile and in-hospital mortality}

Table 2 shows the results of the lipid profile of both groups. In this study, overall, 78.4\% (CI 95\%: 67.3-87.1) of the patients had hypocholesterolemia $(77.6 \%$ and $81.3 \%$ of the survivor and nonsurvivor patients).

The prevalence of hypocholesterolemia in sepsis patients was significantly higher than the Iranian general population (78.4\% vs $8 \%$ ) [95\% confidence interval (CI) of the difference was $59.7-78.3$, $(\mathrm{p}<0.001)]$. Only 2 patients $(2.3 \%)$

Table 1. Baseline clinical features and laboratory investigations of patients $(n=74$, day 0$)$.

\begin{tabular}{|c|c|c|c|c|}
\hline Characteristics & $\begin{array}{c}\text { All participants } \\
(74)\end{array}$ & $\begin{array}{c}\text { Survived } \\
(58)\end{array}$ & $\begin{array}{c}\text { Dead } \\
(16) \\
\end{array}$ & $\mathrm{p}$ \\
\hline Age (year) & $68.0[54.0,81.0]$ & $65.0[53.0,76.0]$ & $80.5[64.5,83.0]$ & $0.025^{*}$ \\
\hline Male $(\%)$ & $40(54.1)$ & $32(55.2)$ & $8(50.0)$ & 0.713 \\
\hline Current smokers (\%) & $11(14.9)$ & $9(15.5)$ & $2(12.5)$ & 0.764 \\
\hline Length of Hospital stay (days) & $9.5[6.0,15.0]$ & $9.5[7.0,14.0]$ & $8.0[2.0,18.5]$ & 0.418 \\
\hline q_SOFA & $36(48.6)$ & $35(60.3)$ & $1(6.3)$ & $<0.001 *$ \\
\hline \multicolumn{5}{|l|}{ Low risk } \\
\hline High risk & $38(51.4)$ & $23(39.7)$ & $15(93.8)$ & \\
\hline \multicolumn{5}{|l|}{ Underlying disease } \\
\hline Diabetes mellitus (\%) & $19(25.7)$ & $18(31.0)$ & $1(6.3)$ & $0.042 *$ \\
\hline Hypertension (\%) & $21(28.4)$ & $17(29.3)$ & $4(25.0)$ & 0.764 \\
\hline Coronary artery disease $(\%)$ & $12(16.2)$ & $11(19.0)$ & $1(6.3)$ & 0.247 \\
\hline Neurologic $(\%)$ & $20(27.0)$ & $16(27.6)$ & $4(25.0)$ & 0.863 \\
\hline Other $(\%)$ & $15(20.3)$ & $8(13.8)$ & $7(43.7)$ & $0.017 *$ \\
\hline Number of underlying diseases & $1.0[0.0,2.0]$ & $1.0[0.0,2.0]$ & $1.0[0.5,1.0]$ & 0.615 \\
\hline Infection site $(\%)$ & & & & 0.117 \\
\hline Respiratory tract & $30(40.5)$ & $22(37.9)$ & $8(50.0)$ & \\
\hline Abdomen & $10(13.5)$ & $9(15.5)$ & $1(6.3)$ & \\
\hline Urogenital tract & $16(21.6)$ & $15(25.9)$ & $1(6.3)$ & \\
\hline Skin/soft tissue & $7(9.5)$ & $6(10.3)$ & $1(6.3)$ & \\
\hline Other infections & $11(14.9)$ & $6(10.3)$ & $5(31.2)$ & \\
\hline Glasgow Coma Scale (GCS) & $14.00(11.00,15.00)$ & $15.00(13.00,15.00)$ & $8.00(6.00,10.50)$ & $<0.001 *$ \\
\hline Systolic blood pressure $(\mathrm{mmHg})$ & $120.0[100.0,130.0]$ & $120.0[100.0,130.0]$ & $102.5[100.0,120.0]$ & 0.083 \\
\hline Diastolic blood pressure (mmHg) & $70.0[60.0,80.0]$ & $80.0[65.0,80.0]$ & $70.0[60.0,80.0]$ & 0.092 \\
\hline Pulse Rate (beats/min) & $109.5[100.0,120.0]$ & $109.5[100.0,120.0]$ & $105.0[96.5,120.0]$ & 0.428 \\
\hline Respiratory rate (breath/min) & $22.0[20.0,26.0]$ & $22.0[20.0,26.0]$ & $27.0[23.0,32.5]$ & $0.001 *$ \\
\hline Temperature $\left({ }^{\circ} \mathrm{C}\right)$ & $38.8[38.0,39.1]$ & $39.0[38.0,39.2]$ & $38.5[37.8,39.0]$ & 0.252 \\
\hline \multicolumn{5}{|l|}{ Laboratory Investigations } \\
\hline Creatinine $(\mathrm{mg} / \mathrm{dL})$ & $1.2[1.0,1.4]$ & $1.1[1.0,1.4]$ & $1.4[1.1,2.2]$ & 0.102 \\
\hline Blood sugar (mg/dL) & $129.0[100.0,167.0]$ & $130.5[99.0,174.0]$ & $122.5[105.0,162.0]$ & 0.622 \\
\hline Bicarbonate (mg/dL) & $22.0[18.9,25.0]$ & $23.2[19.4,25.4]$ & $19.3[15.9,22.3]$ & $0.005 *$ \\
\hline $\mathrm{PaO}_{2} / \mathrm{FiO}_{2}$ ratio & $274.5[196.0,333.0]$ & $291.0[199.0,341.0]$ & $210.0[190.5,294.5]$ & 0.062 \\
\hline White blood cells $(/ \mathrm{mL})$ & $12.4[8.3,15.7]$ & $12.5[8.1,16.8]$ & $12.1[9.2,14.4]$ & 0.641 \\
\hline Poly morphonuclears (\%) & $83.5[78.0,88.0]$ & $83.0[80.0,88.0]$ & $85.0[76.0,88.5]$ & 0.963 \\
\hline \multicolumn{5}{|l|}{ Blood Culture (\%) } \\
\hline Negative & $16(21.6)$ & $10(17.2)$ & $6(37.5)$ & 0.051 \\
\hline Gram-negative bacilli (\%) & $13(17.6)$ & $10(17.2)$ & $3(18.7)$ & \\
\hline Gram-positive cocci (\%) & $4(5.4)$ & $1(1.7)$ & $3(18.7)$ & \\
\hline $\operatorname{ESR}(\mathrm{mm} / \mathrm{h})$ & $36.5[22.0,61.0]$ & $34.0[20.0,61.0]$ & $47.0[28.0,64.5]$ & 0.183 \\
\hline CRP (mg/dL) & $77.0[63.0,89.0]$ & $78.5[63.0,89.0]$ & $70.0[63.5,87.0]$ & 0.660 \\
\hline
\end{tabular}




\begin{tabular}{|c|c|c|c|c|}
\hline Characteristics & $\begin{array}{c}\text { All participants } \\
(74)\end{array}$ & $\begin{array}{c}\text { Survived } \\
(58)\end{array}$ & $\begin{array}{c}\text { Dead } \\
(16)\end{array}$ & $\mathrm{p}$ \\
\hline Total cholesterol (mg/dL) & $124.9 \pm 37.9$ & $125.6 \pm 34.8$ & $122.4 \pm 48.8$ & 0.524 \\
\hline Hypocholesterolemia $(<150)(\%)$ & $58(78.4)$ & 45 (77.6) & $13(81.3)$ & 0.755 \\
\hline Normal (150-199) (\%) & $13(17.6)$ & $11(19.0)$ & $2(12.5)$ & \\
\hline Hypercholesterolemia $(>200)(\%)$ & $3(4.1)$ & $2(3.4)$ & $1(6.3)$ & \\
\hline Triglycerides $(\mathrm{mg} / \mathrm{dL})$ & $114.5[85.0,159.0]$ & $112.5[88.0,151.0]$ & $121.5[80.0,227.5]$ & 0.581 \\
\hline $\mathrm{LDL}-\mathrm{C}(\mathrm{mg} / \mathrm{dL})$ & $67.5[53.0,94.0]$ & $66.5[52.0,95.0]$ & $72.5[61.0,81.0]$ & 0.813 \\
\hline HDL-C (mg/dL) & $22.0[20.0,27.0]$ & $22.0[20.0,27.0]$ & $23.5[20.0,27.5]$ & 0.799 \\
\hline
\end{tabular}

HDL-C $=$ high-density lipoprotein cholesterol, LDL-C $=$ low-density lipoprotein cholesterol

Data are presented as numbers (percentages) or the median [interquartile range]

had $\mathrm{TG}<50 \mathrm{mg} / \mathrm{dL}$ (hypotriglyceridemia). The prevalence of hypotriglyceridemia in sepsis patients was not different from the Iranian general population $(2.3 \%$ vs $4 \%)(15)$.

There were no significant differences between groups in serum levels of STC, LDL-C, HDL-C, and TG. For example, means of the primary STC were $125.6 \pm 34.8$ and $122.4 \pm 48.8$ in survived and deceased levels, respectively $(\mathrm{p}=0.524)$. There were no significant differences between the STC, HDL-C, LDL-C, and TG levels and sex in this study (all $\mathrm{p}>0.05$ ). None of the serum lipids concentrations were associated with length of hospital stay (data not shown).

\section{Lipid profile change and in-hospital mortality}

The mean difference in the absolute change (from baseline to final measurement in $\mathrm{mg} / \mathrm{dl}$ ) of STC, LDL-C, HDL-C, and TG were compared between the 2 groups (the survived against the deceased). The mean difference in reduction of STC, LDL-C, HDL-C, and TG between 2 groups was $25.60 \mathrm{mg} / \mathrm{dL} \quad(\mathrm{p}=0.004), 28.10 \mathrm{mg} / \mathrm{dL}$ $(\mathrm{p}=0.006), 6.90 \mathrm{mg} / \mathrm{dL} \quad(\mathrm{p}=0.010)$, and $37.25 \mathrm{mg} / \mathrm{dL}$ $(\mathrm{p}=0.052)$. The changes in serum lipid levels over time are summarized in Table 3 and Figure 1.

There were positive correlations between clinical outcome of sepsis patients and STC and other lipid profile level changes during hospital stay (not on admission).

\section{Discussion}

Most studies performed to date focused primarily on the comparative analysis of serum lipids between sepsis and severe sepsis groups or between survivor and nonsurvivor groups $(7,16,17)$. In this study, serum lipid levels were analyzed over time, and the association of lipid levels with survival in sepsis patients was also determined.

In this study, the prevalence of hypocholesterolemia in sepsis patients was significantly higher than in the general population. However, absolute STC, LDL, HDL, and TG levels in admission time was not suitable for the prediction of mortality in sepsis patients (based on comparison of survive with nonsurvive groups). The data of this study support the hypothesis that higher decline of STC and other lipid profile during hospital admission are associated with a higher mortality in sepsis patients. Recently, in a study in South Korea, the changes in serum lipid levels over time were examined from the day of admission to ICU (day 0 to day 7). There was a significant gap in the mean value for each lipid and lipoprotein between survivor and non-survivor groups at different time points (18).

Some studies on healthy volunteers injected with a low dose of endotoxin or on septic patients have shown that the systemic inflammatory response is associated with disruption of lipid metabolism $(19,20)$. These changes include reductions in HDL levels and increases in STC and TG (clinically termed the lipaemia of sepsis). On the other hand, some studies revealed that high levels of serum cytokines decrease STC levels during the course of infection, and in critically ill patients, hypocholesterolemia is associated with poor outcomes (21). In this study, comparing the recovered and nonrecovered patient groups, it was found that lipid metabolism was different in the 2 subgroups. Serum lipid concentration in deceased sepsis patients decreased continuously but increased in recovered patients. Changes in lipid profile during sepsis seem to be related to increased cytokines, and excessive cytokine increase may reduce (but not increase) the level of serum lipids. In other words, clinically termed 'the lipaemia of sepsis' is not true in all situations (8). Changes in lipid profile in sepsis may be associated with cytokines level, and their excessive increase may reduce (rather increase) serum lipids level.

Results of this study and similar studies showed that hypocholesterolemia $\mathrm{s}$ is more prevalent in sepsis patients than in the general population; however, the mechanisms are not well delineated (22).

Similar to this study, some studies have shown that in emergency departments, adult patients with sepsis can be rapidly identified as being more likely to have poor outcomes if they have positive quick SOFA (qSOFA) scores $(23,24)$. The qSOFA score was introduced as a rapid bedside clinical score to identify patients with a suspected

Table 3. Results of the lipid profile changes during the hospital stay

\begin{tabular}{lccccccccccccccc}
\hline & $\Delta \mathrm{Ch} 1$ & $\Delta \mathrm{Ch} 2$ & $\Delta \mathrm{ChT}$ & $\Delta \mathrm{HDL} 1$ & $\Delta \mathrm{HDL} 2$ & $\Delta$ HDLT & $\Delta \mathrm{LDL} 1$ & $\Delta \mathrm{LDL} 2$ & $\Delta \mathrm{LDLT}$ & $\Delta \mathrm{TG} 1$ & $\Delta \mathrm{TG} 2$ & $\Delta \mathrm{TGT}$ \\
\hline $\begin{array}{l}\text { Mean difference } \\
(\mathrm{mg} / \mathrm{dL})\end{array}$ & 15.62 & 12.43 & 25.60 & 4.02 & 3.26 & 6.90 & 13.92 & 13.49 & 28.10 & 42.35 & -7.21 & 37.25 \\
P value & 0.072 & 0.116 & $0.004^{*}$ & 0.077 & 0.191 & $0.010^{*}$ & 0.088 & 0.206 & $0.006^{*}$ & 0.207 & 0.642 & 0.052 \\
\hline
\end{tabular}

$0.072 \quad 0.116 \quad 0.004 *$

0.077

cholesterol2 - cholesterol0

$\triangle$ HDL1: HDL1- HDL0, $\triangle$ HDL2: HDL2- HDL1, $\triangle$ HDLT: HDL2- HDL0

$\Delta$ LDL1: LDL1- LDL0, $\triangle$ HDL2: LDL2- LDL1, $\triangle$ LDLT: LDL2- LDL0

$\Delta \mathrm{TG}$ : triglyceride1- triglyceride $0, \Delta \mathrm{TG}$ 2: triglyceride2- triglyceride1, $\Delta \mathrm{TGT}$ : triglyceride2- triglyceride 0

0 : on admission, 1: middle of hospitalization, 2 : before discharge or death 

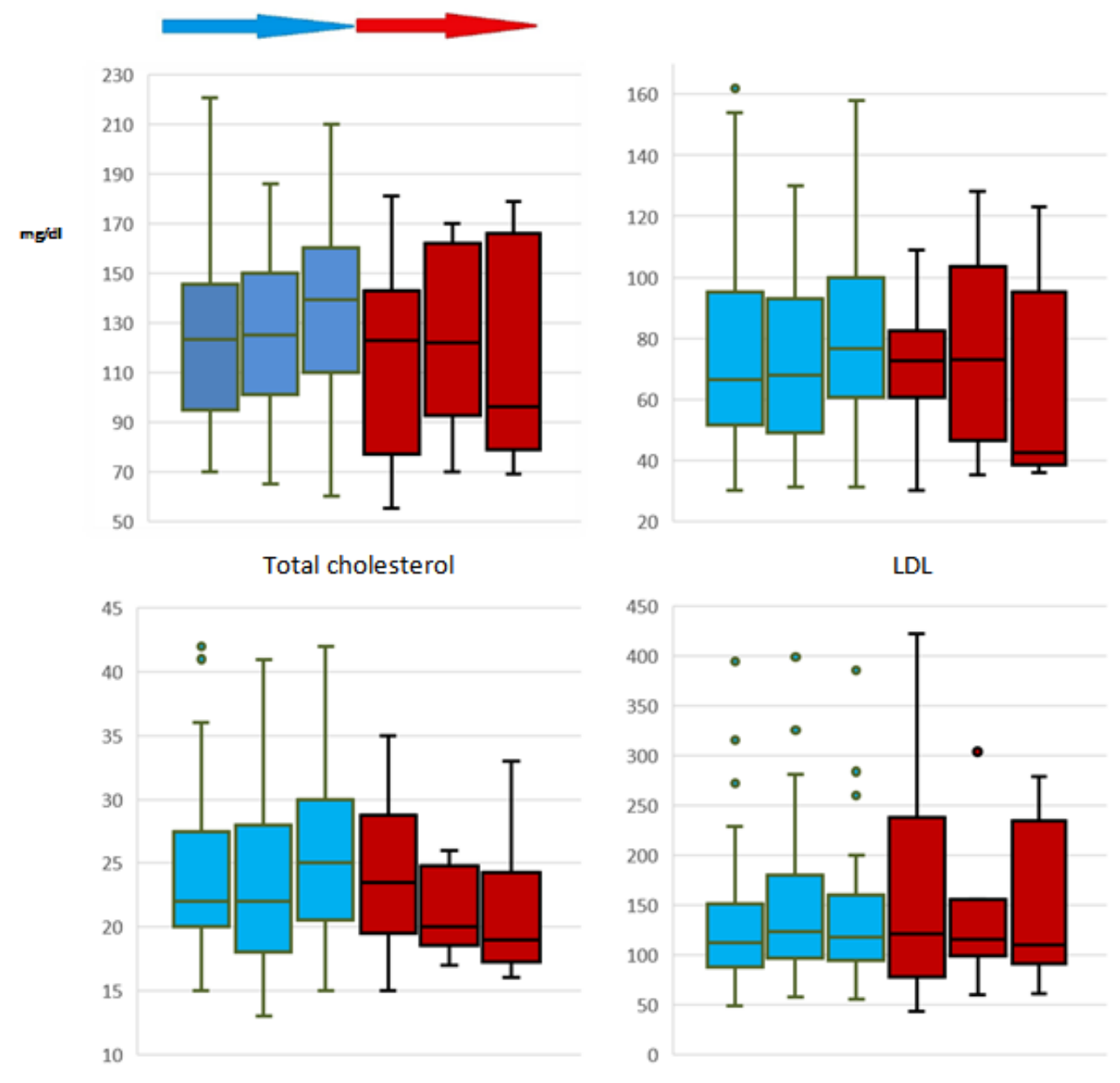

HDL

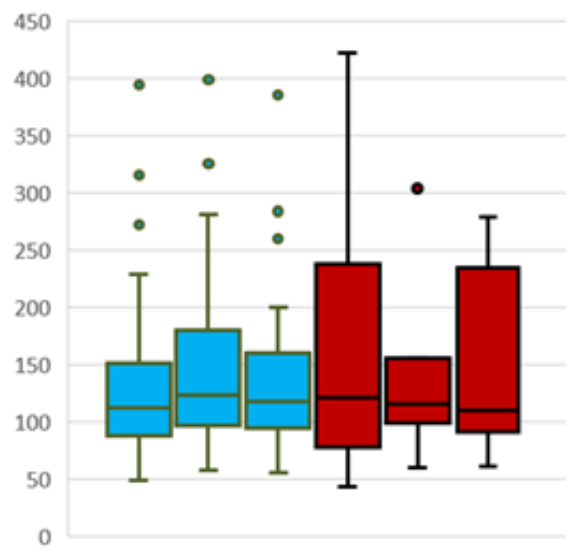

TG

0: On admission, 1: Middle of hospitalization, 2: Before discharge or death, Blue plot: Discharged, Red plot: Deceased.

Fig. 1. Box plot of the serum lipids trends during hospital admission for 2 study groups (discharged and deceased patients)

infection that are at greater risk for a poor outcome (eg, in-hospital mortality predictor) (12). The qSOFA score includes " $\mathrm{RR} \geq 22$ breath/min", "systolic blood pressure $\leq 100 \mathrm{mmHg}$ ", and "altered mental status (Glasgow Coma Scale $<15)$ ]". A "positive" qSOFA Score $(\geq 2)$ suggests high risk of poor outcome in patients with suspected infection (3- to 14-fold increase risk for in-hospital mortality) (23). These patients should be carefully assessed for evidence of organ dysfunction.

There are several pre-existing illnesses that increase susceptibility to development of sepsis and severe sepsis. In this study, history of DM was present in $25.7 \%$ of patients with sepsis. DM alters immunity through several different mechanisms. DM is a common disorder with well-established deleterious effects on immunity and confirmed consequences on the incidence of sepsis or severe sepsis (11).

In this study, Gram-negative isolated bacteria were 3fold higher than Gram-positive organisms. According to the most recent estimates in sepsis, the most common cause of sepsis in developed countries are Gram-positive microorganism. Conversely, the responsible organisms of sepsis in the developing world are more likely to be Gram-negative enteric pathogens (2).

This study had 2 limitations. First, cytokines was not measured, which may help explain the findings. Second, data on the patients' nutrition status before and during hospital admission were not collected. Another limitation of the study was its observational design, although data were collected prospectively.

\section{Conclusion}

This study showed that the variations in lipid profiles differed significantly between the survivor and nonsurvivor groups. Also, more decline of lipid profile was significantly associated with increase of mortality in sepsis patients. However, further studies are required to approve the role of lipid profile in the outcomes of sepsis patients.

\section{Acknowledgments}

We acknowledge the contribution of the many health care workers at the study site who greatly contributed to this study. The present study has been financially supported by the research deputy of Qazvin University of Medical 
Sciences (project number: 14002138).

\section{Conflict of Interests}

The authors declare that they have no competing interests.

\section{References}

1. Weber GF, Chousterman BG, He S, Fenn AM, Nairz M, Anzai A, et al. Interleukin-3 amplifies acute inflammation and is a potential therapeutic target in sepsis. Science. 2015;347(6227):1260-1265.

2. Martin GS. Sepsis, severe sepsis and septic shock: changes in incidence, pathogens and outcomes. Expert Rev Anti Infect Ther. 2012;10(6):701-706.

3. Green P, Theilla M, Singer P. Lipid metabolism in critical illness. Curr Opin Clin Nutr Metab Care. 2016;19(2):111-115.

4. Marik PE. Dyslipidemia in the critically ill. Crit Care Clin. 2006;22(1):151-159.

5. Wendel M, Paul R, Heller AR. Lipoproteins in inflammation and sepsis. II. Clinical aspects. Intensive Care Med. 2007;33(1):25-35.

6. Kruger PS. Forget glucose: what about lipids in critical illness? Crit Care Resusc. 2009;11(4):305.

7. Filippas-Ntekouan S, Liberopoulos E, Elisaf M. Lipid testing in infectious diseases: possible role in diagnosis and prognosis. Infection. 2017;45(5):575-588

8. Yildiz B, Ucar B, Akşit A, Aydogdu SD, Colak O, Colak E. Diagnostic values of lipid and lipoprotein levels in late onset neonatal sepsis. Scand J Infect Dis. 2009;41(4):263-267.

9. Cirstea M, Walley KR, Russell JA, Brunham LR, Genga KR, Boyd $\mathrm{JH}$. Decreased high-density lipoprotein cholesterol level is an early prognostic marker for organ dysfunction and death in patients with suspected sepsis. J Crit Care. 2017;38:289-294.

10. Osborn TM, Phillips G, Lemeshow S, et al. Sepsis severity score: an internationally derived scoring system from the surviving sepsis campaign database. Crit Care Med. 2014;42(9):1969-1976.

11. Hodgin KE, Moss M. The epidemiology of sepsis. Curr Pharm Des. 2008;14(19):1833-1839.

12. Cirstea M, Walley KR, Russell JA, Brunham LR, Genga KR, Boyd $\mathrm{JH}$. The third international consensus definitions for sepsis and septic shock (sepsis-3). JAMA. 2016;315(8):801-810.

13. Segoviano-Mendoza M, Cárdenas-de la Cruz M, Salas-Pacheco J, Vázquez-Alaniz F, La Llave-León $\mathrm{O}$, Castellanos-Juárez $\mathrm{F}$, et al. Hypocholesterolemia is an independent risk factor for depression disorder and suicide attempt in Northern Mexican population. BMC Psychiatry. 2018;18(1):7.

14. Suzuki Y, Sugai T, Fukui N, Watanabe J, Ono S, Tsuneyama N, et al. High prevalence of underweight and undernutrition in Japanese inpatients with schizophrenia. Psychiatry Clin Neurosci. 2014;68(1):78-82.

15. Azizi F, Rahmani M, Ghanbarian A, Emami H, Salehi P, Mirmiran $\mathrm{P}$, et al. Serum lipid levels in an Iranian adults population: Tehran Lipid and Glucose Study. Eur J Epidemiol. 2003;18(4):311-319.

16. Barlage S, Gnewuch C, Liebisch G, Wolf Z, Audebert FX, Glück $\mathrm{T}$, et al. Changes in HDL-associated apolipoproteins relate to mortality in human sepsis and correlate to monocyte and platelet activation. Intensive Care Med. 2009;35(11):1877-1885.

17. Guirgis FW, Dodani S, Leeuwenburgh C, Moldawer L, Bowman J, Kalynych C, et al. HDL inflammatory index correlates with and predicts severity of organ failure in patients with sepsis and septic shock. PLoS One. 2018;13(9):e0203813.

18. Lee SH, Park MS, Park BH, Jung WJ, Lee IS, Kim SY, et al. Prognostic implications of serum lipid metabolism over time during sepsis. Biomed Res Int. 2015;2015.

19. Hudgins LC, Parker TS, Levine DM, Gordon BR, Saal SD, Jiang $\mathrm{XC}$, et al. A single intravenous dose of endotoxin rapidly alters serum lipoproteins and lipid transfer proteins in normal volunteers. $\mathrm{J}$ Lipid Res. 2003;44(8):1489-1498.

20. Bermudes AC, de Carvalho WB, Zamberlan P, Muramoto G, Maranhão RC, Delgado AF. Changes in lipid metabolism in pediatric patients with severe sepsis and septic shock. Nutrition. 2018;47:1049.

21. Murch O, Collin M, Hinds CJ. Lipoproteins in inflammation and sepsis. I. Basic science. Intensive Care Med. 2007;33(1):13-24.

22. Lekkou A, Mouzaki A, Siagris D, Ravani I, Gogos CA. Serum lipid profile, cytokine production, and clinical outcome in patients with severe sepsis. J Crit Care. 2014;29(5):723-727.

23. Raith EP, Udy AA, Bailey M, McGloughlin S, MacIsaac C, Bellomo R, et al. Prognostic accuracy of the SOFA score, SIRS criteria, and qSOFA score for in-hospital mortality among adults with suspected infection admitted to the intensive care unit. JAMA. 2017;317(3):290-300.

24. Freund Y, Lemachatti N, Krastinova E, Van Laer M, Claessens YE, Avondo A, et al. Prognostic accuracy of Sepsis-3 criteria for inhospital mortality among patients with suspected infection presenting to the emergency department. JAMA. 2017;317(3):301-308. 